\title{
Adaptive Waveforms for Target Class Discrimination
}

\author{
Jun Hyeong Bae and Nathan A. Goodman \\ Department of Electrical and Computer Engineering \\ University of Arizona \\ 1230 E. Speedway Blvd, Tucson, Arizona 85721 \\ dolbit@email.arizona.edu; goodman@ece.arizona.edu
}

\begin{abstract}
This paper compares the performance of two matched-illumination waveform design techniques for distinguishing between $M$ target hypotheses. The waveforms are implemented within a closed-loop, sequential-testing framework. In contrast to our earlier work, in this paper the target hypotheses are statistically characterized by power spectral densities. Thus, the waveforms are matched to the target class rather than to individual target realizations. As the class probabilities change in response to received data, the waveforms are adapted, which leads to faster decisions.
\end{abstract}

\section{INTRODUCTION}

Adaptive and knowledge-based (KB) signal processing focus on improving radar performance through advanced signal processing at the receive end of the system. However, rather than develop transmission waveforms and signalprocessing techniques independently, it is useful to consider the implementation and performance of a closed-loop radar system. The closed-loop system possesses an adaptive radar transmitter that responds to the propagation environment and to previously received data. This type of system, termed cognitive radar in [1], can be viewed as an intelligent system that continually interrogates the environment in order to achieve its objectives. Cognitive radar uses prior information, measurements from other sensors, and its own collected data to learn about the environment and make realtime decisions about how to proceed.

In [2], Bell applied information theory to design radar waveforms. He developed optimum waveforms for target detection and information extraction. Optimum detection waveforms focus their energy into one or two frequency bands where the target's reflections are strongest, but optimal information extraction waveforms spread energy over multiple bands where the target ensemble's so-called spectral variance is greatest. In [3], Guerci and Pillai develop a theory for the two-target ID problem and then propose an extension to the $M$-target ID problem. In [3], it is proposed that the average distance between received echoes should be maximized. The problem of temporal waveform shaping for improved target detection and identification is also considered in [4].

In [5-6], we have developed a closed-loop framework for radar target identification when the targets can each be characterized by a known impulse response. In this framework, we use sequential hypothesis testing to determine when the experiment should be terminated while achieving a desired error rate. Two different matched waveforms are compared along with basic wideband and narrowband waveforms. In this work, we relax the assumption of known target impulse responses and instead assume that the target hypotheses are statistically characterized by known power spectral densities (PSD). Therefore, the actual target realization is an unknown sample function generated from the PSD of the true target class. In Section II, we develop the problem statement, and describe the signal model. In Section III, describe the closed-loop operational concept. We describe the general closed-loop framework, use of the sequential testing procedure, and how the system's understanding of the channel can be updated after each data collection. In Section IV, we summarize the two matched waveform approaches that are used. In Section $\mathrm{V}$, we present simulated results, and in Section VI we make our conclusions.

\section{PROBLEM STATEMENT}

It is assumed that there are $M$ channel hypotheses denoted as $H_{1}, H_{2}, \ldots, H_{M}$. Each channel hypothesis is characterized by a power spectral density $\Psi_{i}(f), i=$ $1,2, \ldots, M$. We will employ a real signal model, therefore, the PSDs are symmetric. Let the waveform transmitted by the system be denoted as $s(t)$. When $s(t)$ is transmitted, the received signal can be represented as

$$
y(t)=h(t) * s(t)+n(t)
$$


where * denotes the convolution operator, $y(t)$ is the received signal, and $n(t)$ is additive white Gaussian noise (AWGN) with average power normalized to $\sigma_{n}^{2}=1$.

In order to implement a simulation, it is convenient to have a discrete-time representation of the signal model. In the discrete-time formulation, the transmission waveform is denoted by a length- $L_{s}$ vector $\mathbf{s}$ and the target impulse response is represented by a length- $L_{h}$ vector $\mathbf{h}$. In order to facilitate implementation and manipulation of equations, we define a signal matrix $\mathbf{S}$ as [4]

$$
\mathbf{S}=\left[\begin{array}{ccccc}
s(1) & 0 & \ldots & \ldots & 0 \\
s(2) & s(1) & \ddots & \ldots & 0 \\
\vdots & \vdots & \ddots & \ddots & \vdots \\
s\left(L_{s}\right) & s\left(L_{s}-1\right) & \ldots & s(1) & 0 \\
0 & s\left(L_{s}\right) & s\left(L_{s}-1\right) & \ldots & s(1) \\
\vdots & 0 & s\left(L_{s}\right) & \ldots & s(2) \\
\vdots & \vdots & 0 & \ddots & \vdots \\
0 & 0 & \ldots & 0 & s\left(L_{s}\right)
\end{array}\right]
$$

We can then express the received signal as

$$
\mathbf{y}=\mathbf{S h}+\mathbf{n} .
$$

Next, we let $\alpha_{i, j}$ for $i \neq j$ be the desired probability of incorrectly selecting $H_{j}$ given that $H_{i}$ is true. If multiple transmissions are made, and the data collected on the $k^{\text {th }}$ transmission are $\mathbf{y}_{k}$, then the likelihood ratio for a pair of hypotheses $i$ and $j$ after the $k^{\text {th }}$ data observation is defined as

$$
\Lambda_{i, j}^{k}=\frac{p_{i 1}\left(\mathbf{y}_{1}\right) p_{i 2}\left(\mathbf{y}_{2}\right) \cdots p_{i k}\left(\mathbf{y}_{k}\right)}{p_{j 1}\left(\mathbf{y}_{1}\right) p_{j 2}\left(\mathbf{y}_{2}\right) \cdots p_{j k}\left(\mathbf{y}_{k}\right)}
$$

where $p_{i k}\left(\mathbf{y}_{k}\right)$ is the pdf of the $k^{\text {th }}$ observation under the $i^{\text {th }}$ hypothesis. In sequential hypothesis testing, the experiment is terminated and $H_{m}$ is selected when the condition

$$
\Lambda_{m, j}^{k}>\frac{1-\alpha_{m, j}}{\alpha_{m, j}} \text { for all } j \neq m
$$

is met for some $m$. After a given illumination and data collection, if the condition in (5) is not met, then another transmission is made. The threshold in (5) is the threshold necessary to prevent the average rate of making an error in favor of $H_{j}$ when $H_{i}$ is true from exceeding $\alpha_{i, j}$.

To implement (4), the pdf of the data under each hypothesis is needed. We model the impulse response vector

\section{Closed-Loop Radar}

Interrogate/Illuminate the Channel

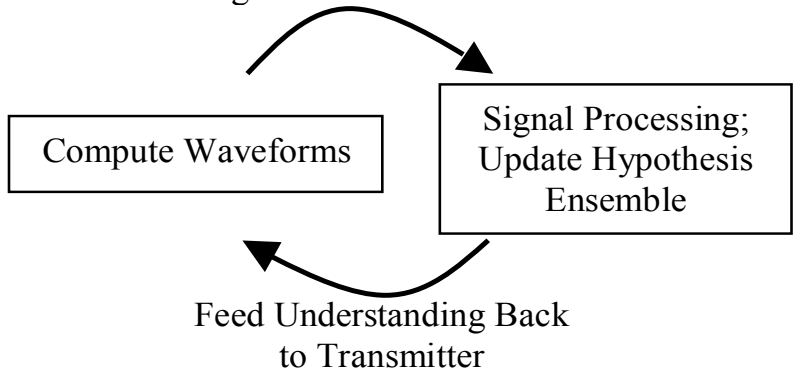

Figure 1. Diagram of closed-loop radar
interrogation with adaptive waveforms.

$\mathbf{h}$ as a Gaussian random vector characterized under the $i^{\text {th }}$ hypothesis by mean vector $\boldsymbol{\mu}_{h, i}$ and covariance matrix $\mathbf{K}_{h, i}$. Since the quantity $\mathbf{S h}$ in (3) is clearly a linear operation on the Gaussian vector $\mathbf{h}, \mathbf{y}$ is the sum of two Gaussian random vectors. Hence, $\mathbf{y}$ is also Gaussian distributed. Under the $i^{\text {th }}$ hypothesis, the mean of $\mathbf{y}$ is $\boldsymbol{\mu}_{y, i}=\mathbf{S} \boldsymbol{\mu}_{h, i}$ and the covariance is $\mathbf{K}_{y, i}=\mathbf{S K}_{h, i} \mathbf{S}^{\mathrm{T}}+\sigma_{n}^{2} \mathbf{I}$. Hence, we have

$$
\begin{aligned}
p\left(\mathbf{y} \mid H_{i}\right)= & \frac{1}{(2 \pi)^{L_{y} / 2}\left|\mathbf{K}_{y, i}\right|^{1 / 2}} \\
& \times \exp \left[-\frac{1}{2}\left(\mathbf{y}-\boldsymbol{\mu}_{y, i}\right)^{\mathrm{T}} \mathbf{K}_{y, i}^{-1}\left(\mathbf{y}-\boldsymbol{\mu}_{y, i}\right)\right]
\end{aligned}
$$

\section{Closed-Loop Operation}

The closed-loop radar interrogation procedure is as follows. First, we transmit a waveform matched to the initial ensemble of class PSDs and collect the resulting data. Once we have the data, we form likelihood ratios and compare to thresholds derived according to the theory of sequential hypothesis testing. If one of the hypotheses meets the criterion on (5), we make a decision in favor of that hypothesis and conclude the experiment. If a decision cannot be made, another round of data collection is required. However, before collecting more data, we update our understanding of the scenario in order to further optimize the transmission waveform. Once a new waveform is computed, another transmission can commence, and the process repeats until a decision is made. The goal of integrating the sequential testing procedure with closed-loop adaptation of the matching waveform is to reduce the number of transmission and energy required to make a decision. Figure 1 shows a diagram of the procedure.

When a decision cannot be made, the system's understanding of the channel must be updated. In this application, the channel update can involve two steps. First, the probabilities associated with each of the hypotheses are 
updated via Bayes' rule. The probability that the $i^{\text {th }}$ hypothesis is true after the $k^{\text {th }}$ observation is then

$$
\operatorname{Pr}\left(H_{i} \mid \mathbf{y}_{k}\right)=\frac{p\left(\mathbf{y}_{k} \mid H_{i}\right) \operatorname{Pr}\left(H_{i}\right)}{p\left(\mathbf{y}_{k}\right)}
$$

The denominator in (7) is common to each hypothesis and need not be calculated. Instead, after updating each hypothesis, the probabilities are scaled such that their sum is one. Also, the value $\operatorname{Pr}\left(H_{i}\right)$ is the class probability prior to taking the $\mathrm{k}^{\text {th }}$ observation. In the proposed sequential procedure, we set $\operatorname{Pr}\left(H_{i}\right)=\operatorname{Pr}\left(H_{i} \mid \mathbf{y}_{k-1}\right)$, which effectively accounts for all data collected prior to the $k^{\text {th }}$ observation. The matched waveforms that we implement depend on the class probabilities; hence, as the probabilities change, so does the waveform matching. Furthermore, note that the pdf of the received data shown in (6) depends on a data covariance matrix, which in turn depends on the class covariance matrix and transmitted signal through the relationship

$$
\mathbf{K}_{y, i}=\mathbf{S} \mathbf{K}_{h, i} \mathbf{S}^{\mathrm{T}}+\sigma_{n}^{2} \mathbf{I} .
$$

This dependence means that the data pdf changes at each iteration as the transmission waveform is updated.

A second potential way to update the system's understanding of the channel is to consider how the received data affect the system's understanding of the actual target realization. Prior to collecting data, the target classes are characterized by the mean and covariance of their impulse response. It may be possible to update the mean and covariance of each hypothesis in response to the received data. For example, for a Gaussian data model, the sequential linear minimum mean square estimator (LMMSE) provides a method for updating a Bayesian parameter vector. Allowing the signal contribution of the data vector in (3) to be the parameter vector, we can update the mean and covariance of each class. Since the class covariance matrix is related to the variance of its frequency spectrum, these updates may allow improved waveform matching as the actual target realization is determined.

Unfortunately, a couple difficulties exist when trying to implement this second approach. One difficulty is that the statistics of the impulse responses are needed for waveform matching while the sequential LMMSE procedure updates the received signal vector, Sh. With knowledge of transmitted waveforms, however, this difficulty can be overcome. In initial testing, a more significant difficulty lies in the assumption that the sequential LMMSE makes about the accuracy of the data statistics. The LMMSE update equations are based on the assumption that the data mean and covariance is accurate. However, assuming the target hypotheses are distinct, this can only be true for one hypothesis. For this hypothesis, refined estimates of the target realization are viable. For the other hypotheses, the estimated target realizations must be updated with data generated from a different statistical distribution. In time, every hypothesis will begin to believe the data despite the initial class statistics. If a decision has not been made, it is possible to reach a state where the updated mean and covariance of all hypotheses are identical, thus making a decision impossible.

The above issues are still being investigated. Therefore, in this paper we focus on adapting waveforms via updates on the class probabilities. As certain classes are ruled out or appear unlikely, it is less important to distinguish them, and the waveform is allowed to adapt.

\section{WAVEFORM DESIGN}

We apply two different matched waveform techniques. The first techique is based on maximizing mutual information as originally described in [2]. The second technique is based on SNR considerations as described in [34]. Both techniques have previously been used in [5-6] to discriminate between known impulse responses. We now briefly describe how we have applied the techniques to targets characterized by a PSD.

In the information-based approach, we assume that the target is characterized by an impulse response that is a realization of a Gaussian random process. In this case, the time-limited, energy-constrained waveform that maximizes the mutual information between the received signal and the target ensemble has a frequency power spectrum given by [2]

$$
|S(f)|^{2}=\max \left[0, A-\frac{P_{n n}(f) T}{2 \sigma_{H}^{2}(f)}\right] d f
$$

where $\sigma_{H}^{2}(f)=\mathrm{E}\left\{|H(f)-\mathrm{E}\{H(f)\}|^{2}\right\} \quad$ is called the ensemble's spectral variance. The variable $A$ is used to implement the finite energy constraint. In [5-6], the ensemble was defined to be the suite of known impulse responses that characterized the target alternatives. As the hypothesis probabilities evolved, it was simple to form a weighted spectral variance.

In the current scenario, each hypothesis is itself made up of an ensemble of possibilities. For zero-mean Gaussian target realizations, the spectral variance of a single hypothesis is the corresponding PSD, $\Psi_{i}(f)$. To match the waveform to the set of target hypotheses, we form the weighted average PSD according to 


$$
\sigma_{H}^{2}(f)=\sum_{i=1}^{M} \operatorname{Pr}\left(H_{i}\right) \Psi_{i}(f) .
$$

Since (9) implements the waterfilling operation, this waveform approach is referred to as the waterfilling waveform.

The second waveform approach is based on the SNRmaximizing approach of [3-4]. In the case of a two-target ID problem, SNR is maximized by maximizing the distance between the received echoes from the two targets. The solution is based on the autocorrelation of the signal that results from subtracting the two impulse responses. In the present case, this difference signal is random, but its expected value can be obtained from the relationship between the PSD and autocorrelation functions of random processes. Letting the autocorrelation matrix obtained from the difference between hypotheses $i$ and $j$ be $\Omega_{i, j}$, we account for the need to discriminate between all pairs of hypotheses by forming the quantity

$$
\mathbf{Q}=\sum_{i=1}^{M} \sum_{j=i+1}^{M} \operatorname{Pr}\left(H_{i}\right) \operatorname{Pr}\left(H_{j}\right) \Omega_{i, j} \Omega_{i, j}^{\mathrm{T}} .
$$

The matched waveform is taken as the principal eigenvector of $\mathbf{Q}$ normalized to have the required energy. This waveform is referred to as the eigen-based waveform, or the eigensolution.

\section{Results}

We compared the performance of three different waveforms for distinguishing the hypotheses. One waveform was a wideband waveform that was not matched to the target ensemble. The other two waveforms were the waterfilling waveform and the eigen-based waveform. We simulated a scenario with four distinct target classes. Each hypothesis was a Gaussian random processes with known PSD. For each simulation, we generated 2000 different realizations of the true impulse response, and for each realization, the true target hypothesis was randomly selected from the four possibilities.

The desired error rate was $\alpha=0.01$; however, in the case where target classes are not mutually exclusive, it is difficult to actually achieve the desired error rate. We took two measures to handle this. First, the four different class PSDs were defined such that most of their energy were in nonoverlapping bands. This minimized the number of target realizations, or sample functions, whose frequency spectrum actually resembled the PSD of an incorrect target class more than the PSD of the correct target class. Second, in some cases we adjusted the sequential testing thresholds to get error rates reasonably close to the desired rate.

We averaged the number of illuminations required to terminate the sequential test, and also calculated the error rate. Both metrics were computed as a function of the

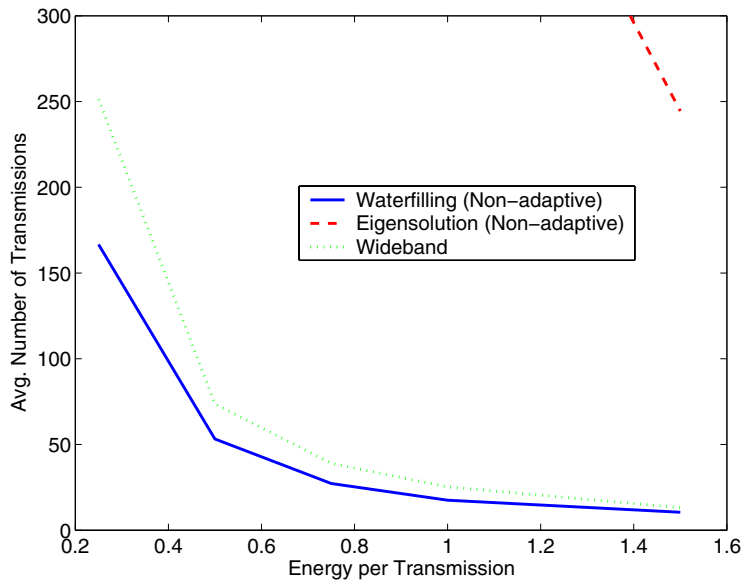

Figure 2. Average transmissions for the non-adaptive approach.

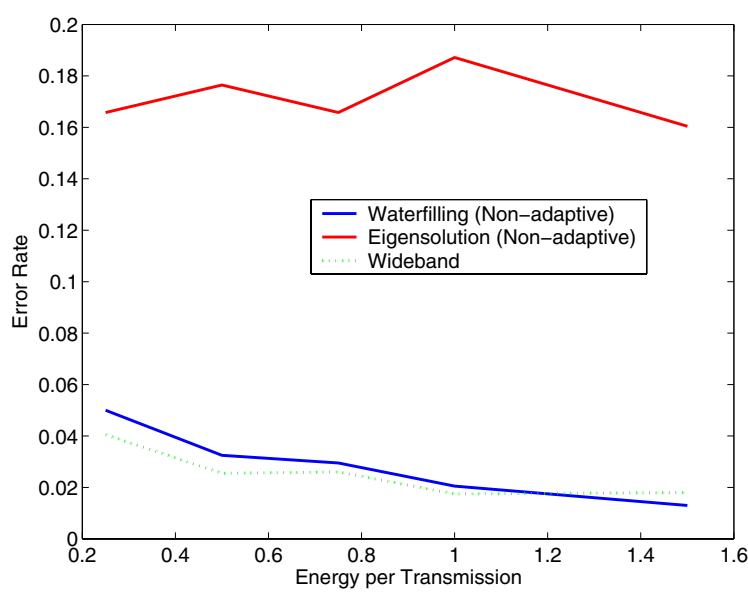

Figure 3. Error rate for different waveforms in the non-adaptive approach.

Figure 2 shows the average number of illuminations to reach a decision when no waveform adaptation is performed. The two matched waveforms are matched to the initial ensemble with equal prior probabilities, but are not adapted as the probabilities change. The waterfilling approach performs best because it is matched to the ensemble but also tends to spread its energy into multiple frequency bands. The eigensolution performed the worst. Even though the eigensolution is a matched approach, it tends to focus its energy into only one or two narrow frequency bands. What happens in this non-adaptive implementation is that if those narrow bands are aligned with the frequency spectrum of the true target realization, little target energy is received. In the non-adaptive scenario, the waveform is not allowed to correct itself as the probabilities change. Figure 3 shows the corresponding error rates for the non-adaptive case. 


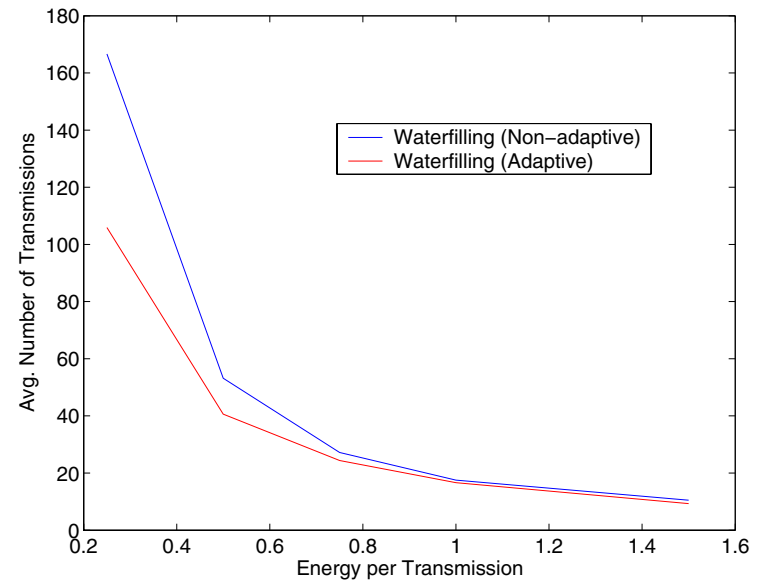

Figure 4. Performance improvement when waterfilling waveform adapts to changing hypothesis probabilities.

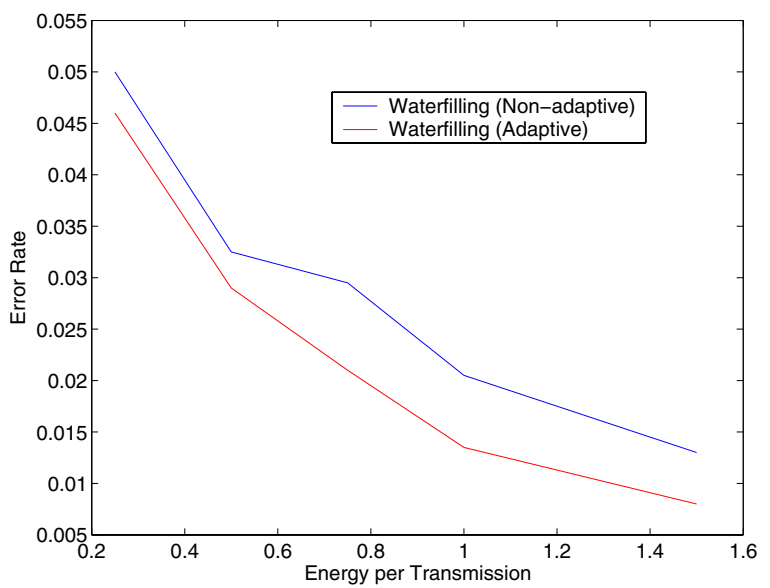

Figure 5. Error rates for adaptive vs. non-adaptive waterfilling waveform implemenation.

Figures 4 and 5 compare results when the waterfilling waveform is used in the non-adaptive, open-loop fashion of the previous figures versus the proposed closed-loop operation complete with an adaptive transmit waveform.
When the waveform is allowed to adapt to its changing understanding of the hypotheses, the required number of transmissions is reduced. This is true even though the closed-loop operation resulted in a slightly lower error rate as the non-adaptive operation.

\section{CONCLUSIONS}

We have tested two different radar transmission waveforms in a sequential testing procedure for identifying statistically characterized target classes. We have discussed several issues that were not important for our previous work with known target impulse responses, but now must be considered. These issues include modification of waveform matching techniques to PSD information as well as issues regarding how to update the system's understanding of the channel. Future work will attempt to better control error rate in these situations, will refine the adaptive implementation of the eigensolution waveform, and will investigate methods of exploiting estimates of the actual target realization in the adaptive waveform matching process.

\section{REFERENCES}

[1] S. Haykin, "Cognitive radar: a way of the future," IEEE Sig. Proc. Mag., vol. 23, no. 1, pp. 30-40, Jan. 2006.

[2] M.R. Bell, "Information theory and radar waveform design," IEEE Trans. Info. Theory, vol. 39, no. 5, pp. 1578-1579, Sept. 1993.

[3] J.R. Guerci and S. U. Pillai, "Theory and Application of Optimum Transmit-Receive Radar," IEEE 2000 International Radar Conference, Washington, DC, pp. 705-710, May 8-12, 2000.

[4] D.A. Garren, M.K. Osborn, A.C. Odom, J.S. Goldstein, S.U. Pillai, and J.R. Guerci, "Enhanced target detection and identification via optimised radar transmission pulse shape," IEEE Proc. Radar, Sonar and Navigation, 148(3), pp. 130-138, June 2001.

[5] N.A. Goodman, P.R. Venkata, and M.A. Neifeld, "Adaptive waveform design and sequential hypothesis testing for target recognition using cognitive radar," to appear in IEEE J. Selected Topics in Sig. Processing, 2007.

[6] P.R. Venkata and N.A. Goodman, "Novel iterative techniques for radar target discrimination," 2006 International Waveform Diversity and Design Conference, Lihue, HI, Jan. 2006. [Cd-Rom]

[7] T.M. Cover and J.A. Thomas, Elements of Information Theory. New York: Wiley, 1991. 